American Journal of Applied Sciences 9 (7): 1107-1112, 2012

ISSN 1546-9239

(C) 2012 Science Publications

\title{
An Alternative Graph Cut Algorithm for Morphological Edge Detection
}

\author{
P. Radhakrishnan \\ Department of Computer Science, College of Computer Science, \\ King Khalid University, P.O. Box: 394, Abha, Kingdom of Saudi Arabia
}

\begin{abstract}
Problem statement: Image analysis has some basic importance of edge detection. Characterize the object boundaries are helpful for object segmentation, registration and identification in a scene. There are many methods for edge detection, but it can be identified by two major methods, search-based and zero-crossing based. Approach: Proposed method tries to follow searching the edge points by applying graph cuts in the place of morphological approach. The graph cut edge detection algorithm is very effective to detect edges with minimum searches. Results: The new approach produces connectives edge boundary with recursive approach in short duration comparatively with morphological approach. It is obtained by identifying the edge points with cut measure within its boundary. Conclusion: The graph cut algorithm detect boundary for any shapes with normal structure, this identification of edges in a complex background is always required a lot of user inputs. In each level only the local neighboring boundaries to be separated with labels. It is optimized approach for unknown region boundaries in complex basins.
\end{abstract}

Key words: Graph cuts, Edge detection, Morphology, Pattern spectrum

\section{INTRODUCTION}

Morphological operations are frequently used for edge identification. In recent years the graph cuts become more user friendly approach for edge detection. The graph cut approach normally converts as undirected graphs, arcs and nodes are considered with adjacency relationship between pixels. Falcao et al. (2006) approach computes an ordered region growing where the propagation order of each pixel is proportional to the cost of an optimum path from the seed set to that pixel. In their method each pixel defines a region which includes it and all pixels with lower propagation order. Also the boundary of each region is a possible cut boundary whose cut measure is also computed an assigned to the corresponding pixel on the fly. Xu et al. (2003) prosposed a method which is a combination of active contours and the optimization tool of graph cuts and differs fundamentally from traditional active contours in that it uses graph cuts to iteratively deform the contour. They easily extends to the segmentation of three and higher dimensional objects. Bai (2010) proposed a novel approach for noise removal cum edge detection for both gray scale and binary images using morphological operations. It demonstrates that the proposed filter cum edge detector approach overcomes the deficiency of conventional methods and efficiently removes the noise and detects the edges. Sagar et al. (2003) proposed a method to extract ridge and valley connectivity networks from a simple DEM as well as a simulated fractal DEM, using non-linear morphological transformations in a methodical way. Stawiaski et al. (2008) applies minimal surfaces and markov random models to the segmentation of liver tumors by using a region graph instead of a pixel graph. Soille and Vogt (2009) presents a method for segmenting binary patterns into seven mutually exclusive categories; core, islet, loop, bridge, perforation, edge and branch. They achieved by applying a series of morphological transformations such as erosions, geodesic dilations, reconstruction by dilation, anchored skeletonisation. Sumengen and Manjunath (2006) introduces new type of variational segmentation cost functions and associated active contour methods that are based on pair wise similarities or dissimilarities of the pixels. Also extens a new curve evolution framework, the graph partitioning active contours. Paulo et al. (2009) explains exploit similar image graphs and some comparative analysis are given. Also gives image segmentation can be elegantly solved by optimum-path forest and minimum cut in graph. They clarify their differences and provide their comparative analysis from the theoretical point of view, for the case of binary segmentation in which hard 
constraints (seeds) are provided interactively. Peng et al. (2011) presented an iterated region merging-based graph cuts algorithm which is a novel extension of the standard graph cuts algorithm. Our approach searching the edge points by calculating edge strength. Then applying the dilation process which generating the adjacency of the current boundary contour and identifying local minima from the contour.

\section{MATERIALS AND METHODS}

Morphological approach: The morphological reconstruction for characterizing shapes and size (Radhakrishnan, 2005) having importance in structuring elements like square, rhombus and circle. Morphological operations have more futures to detect the edges from regular and irregular shapes. The pattern spectrum of size $\mathrm{n}$ by applying reconstruction process with morphological operation and structuring elements like opening of $\mathrm{n}-1$ recursive procedure produces area of shape. The pattern spectrum (Eq.1) is as defined as:

$$
\begin{aligned}
& \operatorname{PS}(\mathrm{n})=\text { Area }\{\text { XonB } / \text { Xo }(n+1) B\} \\
& \text { Where } \mathrm{n}=0,1, \ldots \mathrm{N}_{\text {Max- } 1} \\
& \operatorname{PS}(\mathrm{n})=0, \mathrm{n} \geq \mathrm{N}_{\text {Max }-1}
\end{aligned}
$$

Where $\mathrm{X}$ is the image and $\mathrm{B}$ is the structuring element, $\mathrm{I} \backslash \mathrm{O}=\{\mathrm{x} \in \mathrm{I}: \mathrm{x} \in \mathrm{O}\}, \mathrm{N}_{\max }$ is the minimum size of the $\mathrm{B}$, such that the morphological operation erosion of image $X$ with $N_{\max }$ B results in the empty set. Then the $n^{\text {th }}$ entry in the process is the number of elements of the set difference between the opening of the image X by B of size $\mathrm{n}$ and $(\mathrm{n}+1)$. The morphological opening removes the portion smaller than the SE, the difference of images opened by the SE size $\mathrm{n}$ and next contains the portion whose size is exactly $\mathrm{n}$. Thus the following figure (Fig 1) explains the procedure of pattern spectrum of square using octagon SE in iterative procedure for $\mathrm{n}-1$ openings.

Graph cuts algorithm for edge detection: Edge can be detected using graph cut algorithm, which is better than the morphological approach. The edge which is derived from graph cut algorithm produces minimum of complete structure. The image segmentation detects the boundaries of Edge Neighborhood (EN). The user can specify the EN size using its characteristics of neighbors. So very close edges can be identified which yields from EN structure. At every step of iteration the
EN replaces with its closest edge points of neighbors. Morphological dilation process may extend the size of structure using various structuring elements. In graph cut algorithm the dilation process make the edge closer of structure which mostly required in any form of image. The inner boundary and outer boundary of edge neighborhood segmented through graph cut algorithm. The adjacency graph of EN uses both inner and outer boundary by considering sinking structure and source structure. The final boundary should produce the inner and outer boundaries.

Let a connected graph $\mathrm{G}=(\mathrm{N}, \mathrm{A})$ be a connected graph (Xu et al., 2003) with vertex set $\mathrm{N}$ and edge set $\mathrm{A}$. Each $\operatorname{arc}(\mathrm{u}, \mathrm{v}) \in \mathrm{A}$ has a postive capacity $\mathrm{c}(\mathrm{u}, \mathrm{v}) \geq 0$. If $\operatorname{arc} \notin \mathrm{E}$, we assume that $\mathrm{c}(\mathrm{u}, \mathrm{v})=0$. Two vertices in $\mathrm{N}$ are distinguished: a source $\mathrm{s}$ and a compresed t. A cut $(S, T)$ of the network $G$ is a partition of $\mathrm{N}$ into $\mathrm{S}$ and $\mathrm{T}=\mathrm{N}-\mathrm{S}$ such that $\mathrm{s} \in \mathrm{S}$ and $t \in T$. The limitation of a reduce form is defined as the addition of the all values of the edges across the cut, the $\mathrm{s}-\mathrm{t}$ minimum cut problem is to find a cut in graph $G$ that separates $s$ and $t$ with the smallest capacity. Its more revlavent to graph theory.

The relationship between flows and cuts in networks is very high in transit, low in distraction the For-Fulkerson Theorem (Xu et al., 2003). The various nodes which are taken in account are separated from sink nodes after the process. The same process is very important in our study to reduce the number of nodes and make it closer of edges from the given structure by applying graph cut algorithm. In the process, we try to decrease the number of nodes from the assumption by the way the $n$ number of nodes will be decreased to one or very less number in the second stages. This will delete the self loops of nodes by the parallel edges are merged as shown in Fig. 2. In terms of this operation, we have the following Theorem for the multi-source multi-sink s-t minimum cut problem (Xu et al., 2003).

The vertex of graph is assumed from each pixel of image $\mathrm{v} \in \mathrm{N}$. The adjacent vertexes are connected as arc. The weight of arc is assumed from the similarity between the vertexes. Each boundary of the image separates the image into two parts $\mathrm{S}$ and $\mathrm{T}$ on the structure. The whole minimum among all cuts on the structure considered for reduced form of image with minimum vertex. The edge neighborhoods are detected using reduced form of image. The initial arcs are replaced with minimum cuts to get the edge neighborhoods. 

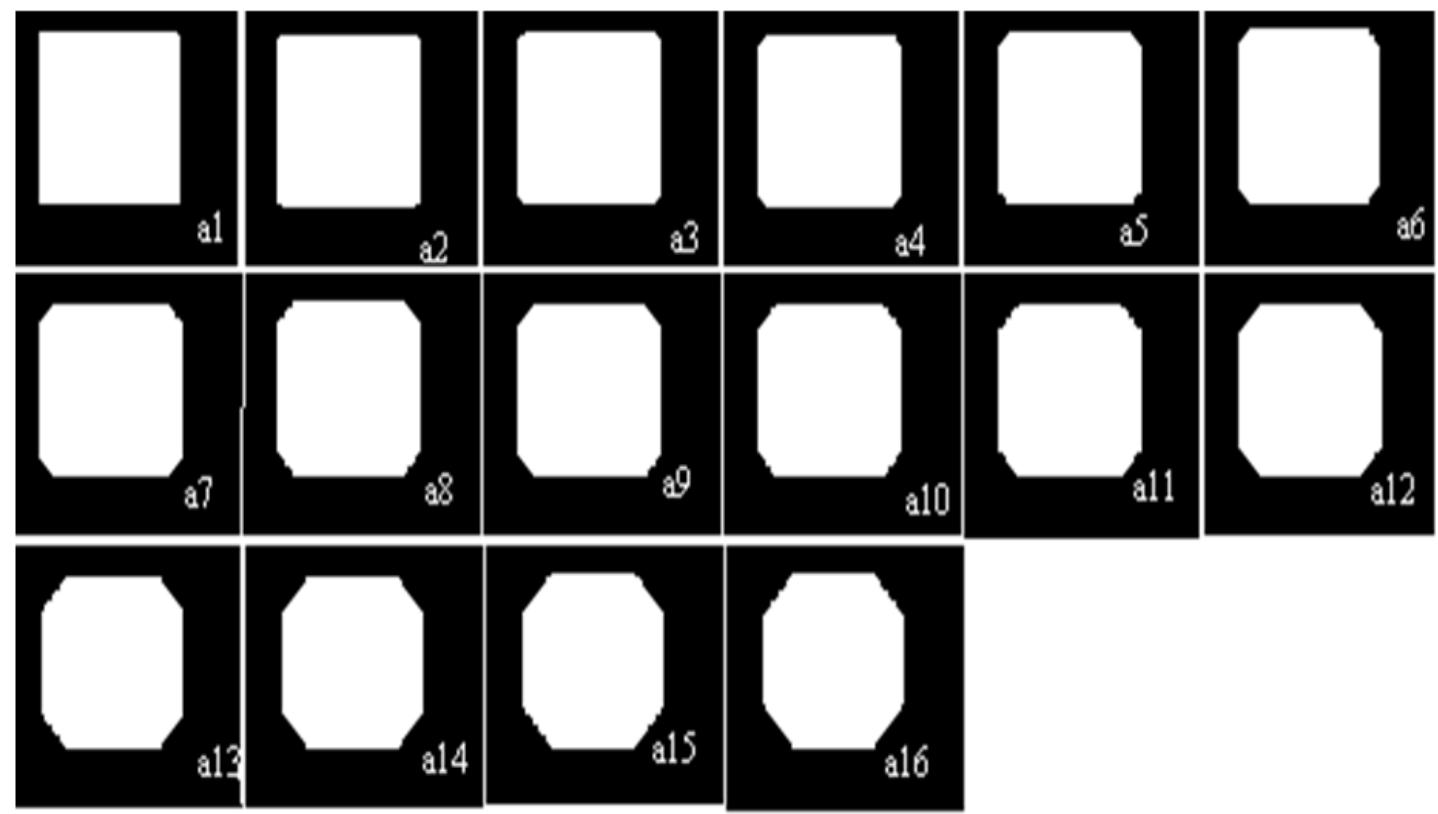

Fig. 1: Morphological operations on Pattern spectrum of square with octagon as structuring element. a1: Image with one opening, a2-a15 different scale openings, a16: Image with n-1 openings
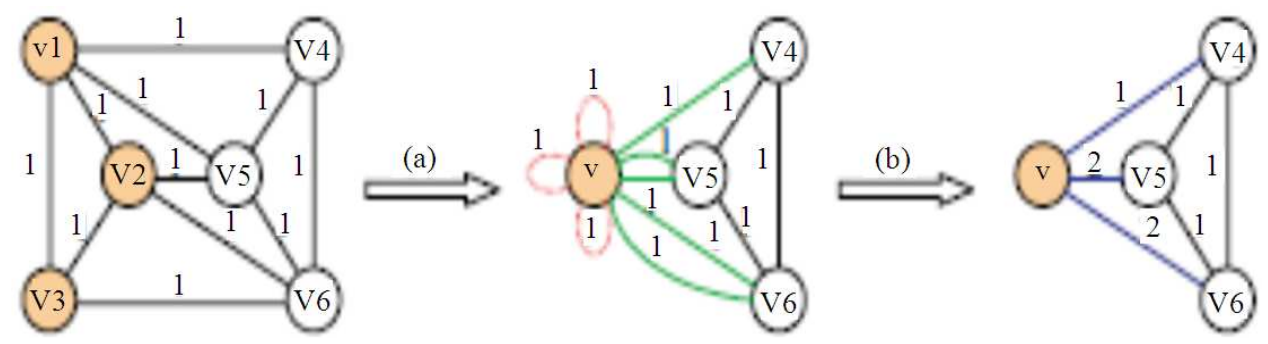

Fig. 2: Node identification (Xu et al., 2003) (a) v1, v2, v3 are joined and created node v. (b) Inner loops are removed and similar arcs are changed by a one arc.

The following steps are followed for the graph cut method instead of morphological approach:

- Identify the adjacency graph of the given structure.

- Produce the edge neighborhoods by dilating the boundary of inner and outer. (Fig. 2)

- Consider the pixels of inner structure as one source and identify the pixels corresponding to the extreme outer boundary as sink structure.

- Find the minimum cut obtain new structure by separates inner and outer.

- Go to step 2 until get the minimum structure pixels.

Initially the dilation process identifies the adjacency structure with pixels of assumed image; this is capable of converting the whole structure into minimum of edge neighborhoods. Secondly, if the neighborhoods are homogeneous, the whole optimal structure must be inner contour. The corresponding graph will have $\mathrm{n}$ arcs as least number by cut algorithm. Next the dilation produces an inner structure that is from multiple sources of graph. The sources are single which parts of the s-t minimum cut are.

\section{RESULTS}

Figure 3 shows an image with gray representation, rhombs object constructed to identify the strength of algorithm to initial structure. The sequence of images correspond the use of various source contours. The leftmost image in each row shows the first structure and last one shows the determined the boundary. The structures which are placed in middle represent the intermediate steps of algorithm. 


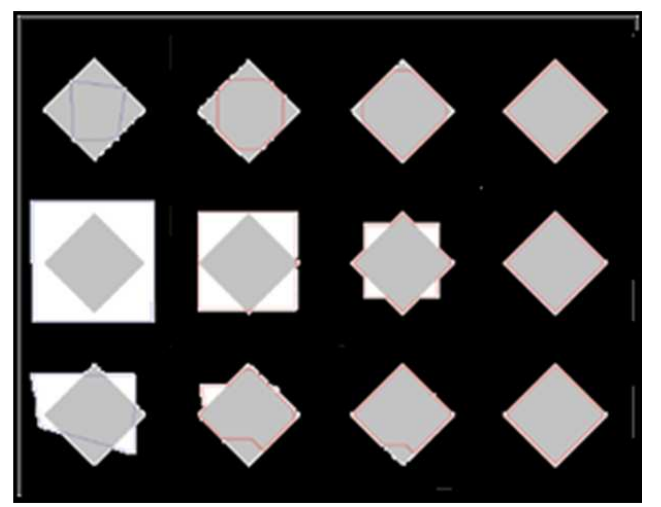

Fig. 3: initial contour are represented in the leftmost image of each row. The deformationof contour explained in interved images.

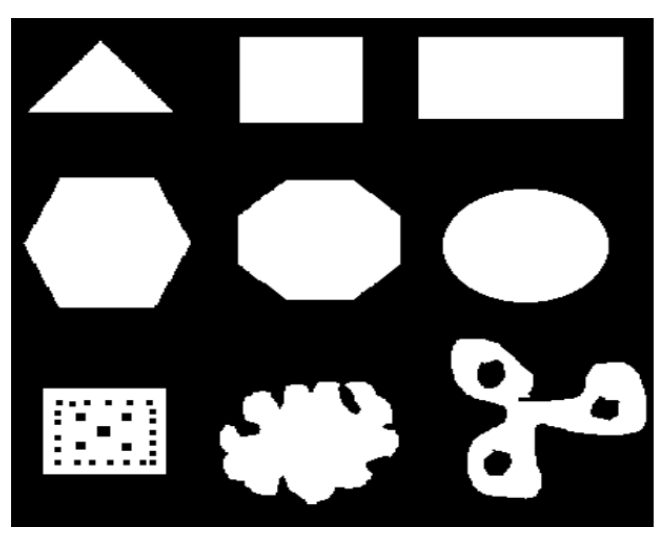

Fig. 4: Sample Shapes considered for study of Edge detection using Graph cuts

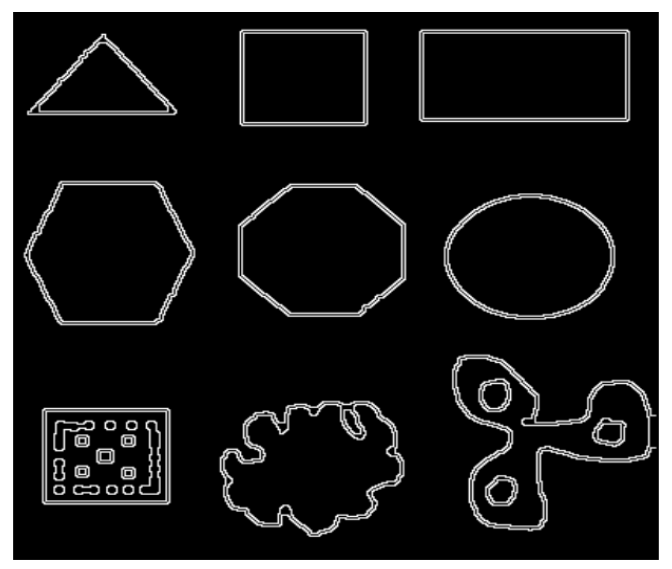

Fig. 5: Edge neighborhoods are identified by using dilation process from the initial contour and outer contour. The initial and final vertices are used for inner and outer boundaries of the corresponding shape

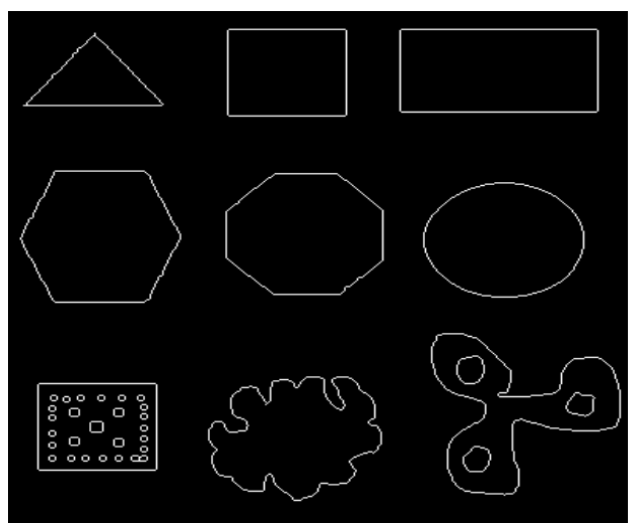

Fig. 6: The final edges resulting from our approach is given with its Edge Neighborhoods
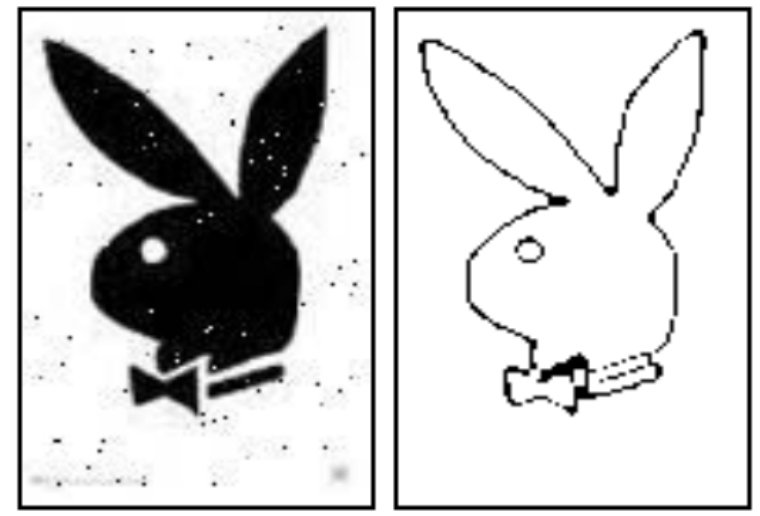

Fig. 7: The left rabbit figure is considered and we got the result of edges by applying graph cut method shown in right side

By applying Graph cut algorithm for edge detection of the shapes given in Fig. 4, we got the results first with multiple counters as given Fig. 5. The multiple counters are inner and outer of the given shapes shown in Fig. 5.The connectivity graph produces the inner and outer structure from initial and reduced number of vertices.

Using the inner and outer counters we derive the final counter as edges of given shapes by applying the minimum cut methods. These edges are more suitable for shapes, to know the difference of various shapes; we considered the square, octagon, triangle, circle, rectangle and irregular shapes with different partitions. We can easily see the exact edges for the difference shapes from the results shown in Fig. 6. The same approach we applied in rabbit Fig. 7 left and the result shown in right side. Similarly we applied in watershed given Fig. 8. 


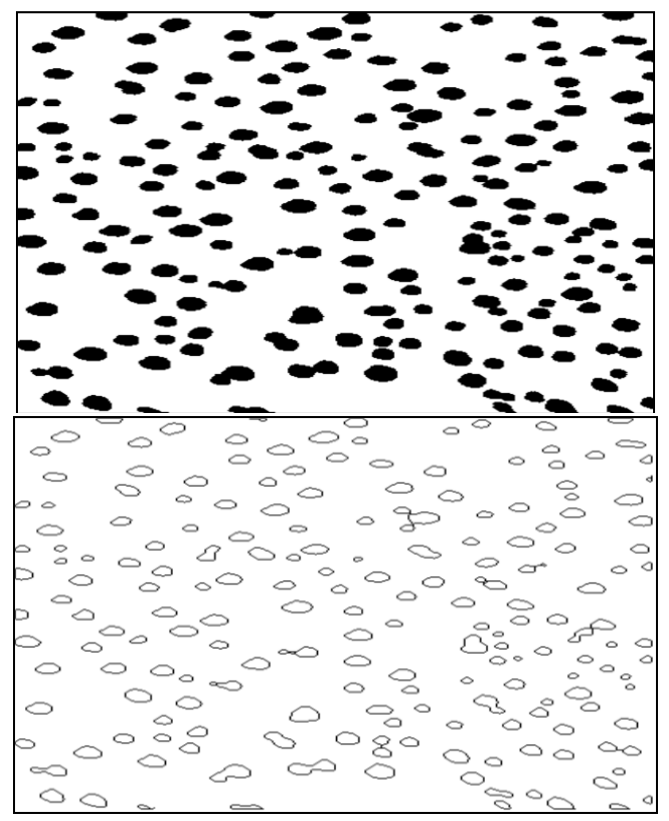

Fig. 8: Another example of watershed is considered given in top and we got the result of edges by applying graph cut method shown in bottom

\section{DISCUSSION}

The graph cuts algorithm produces the changes in edges on each step, which are more deformed edges starts from the beginning. Global form of final edges derived from the specified region of image which has a differentiation of background. A morphological approach mostly reduces or dilates by applying operations like opening or closing in sequences. But graph cut algorithm continuously produce the reduced number of vertices and removing the self loop of each node by considering the adjacency of graph. If the final contour is not in required form, the operators can change the vertex or arc of adjacency iteratively. The inner and outer boundaries are helpful for the deformation of edges from initial structure.

The multi-source and multi-sink neighborhoods of edges will try to find the closest edges of given image. These sources are derived from our algorithm by introducing the graph cut methods. The multi-source produces the difference of resultant structure. The multi-sink makes the edge points with close contrast. The 8-connectivity produces smoother structures than the 4-connectivity graph. The boundary regions are measured and identified the adjacency of vertex corresponding to pixel locations.

\section{CONCLUSION}

Interactive approach allows the user to select the region which has to focus for detect the edges by applying our approach. The infinite number vertex in the given region will be converged as finite number of pixels by sinking the adjacency of graph.

The future problems relevant to this study will be incorporating the texture and color details of image for detecting the exact edges. Edge neighborhoods made the optimal area of structure from the given form to reduced form without losing the important information.

The traditional morphological approach try to increase or decrease area by loosing or appending some information. But the graph cut try to apply the logical for sinking the vertex set with arcs. This helps to produce the appropriate structure of edges from the input image. It is more suitable for considering the less computation time for identifying the edges of given structure.

\section{REFERENCES}

Sagar, B.S.D., M.B.R. Murthy, C.B. Rao and B. Raj, 2003. Morphological approach to extract ridge and valley connectivity networks from digital elevation models. Int. J. Remote Sensing, 24: 573-581.

Falcao, A.X., P.A.V. Miranda and A. Rocha, 2006. A linear-time approach for image segmentation using graph-cut measures. Adv. Concepts Intelligent Vision Syst. Lecture Notes Comput. Sci.,

Paulo, A., V. Miranda and X. Alexandre, 2009. Falcão links between image segmentation based on optimum-path forest and minimum cut in graph. J. Math. Imaging Vision, 35: 128-142.

Peng, B., L. Zhang, D. Zhang and J. Yang, 2011. Image segmentation by iterated region merging with localized graph cuts. Patt. Recog., 44: 2527-2538. DOI: 10.1016/j.patcog.2011.03.024

Radhakrishnan, P., 2005. Characterization of reconstructed basins using pattern spectrum procedure. Am. J. Applied Sci., 2: 843-846. DOI: 10.3844/ajassp.2005.843.846

Bai, M.R., V. Venkata Krishna and J. SreeDevi, 2010. A new Morphological Approach for Noise Removal cum Edge Detection. Int. J. Comput. Sci. Iss., 7: 1694-0814.

Soille, P. and P. Vogt, 2009. Morphological segmentation of binary patterns. Patt. Recog. Lett., 30: 456-459. DOI: 10.1016/j.patrec.2008.10.015 
Stawiaski, J., E. Decencière and F. Bidault, 2008. Interactive liver tumor segmentation using graphcuts and watershed. Proceedings of the 11th International Conference on "Medical Image Computing and Computer Assisted Intervention, (MICCAI' 08), France, pp: 1-12.

Sumengen, B. and B.S. Manjunath, 2006. Graph Partitioning Active Contours (GPAC) for image segmentation. IEEE Trans., 28: 509-521. DOI: 10.1109/TPAMI.2006.76
Xu, N., R. Bansal and N. Ahuja, 2003. Object segmentation using graph cuts based active contours. Proceedings of IEEE Computer Society Conference on Computer Vision and Pattern Recognition, (CVPR' 03), IEEE Xplore Press, USA, $\quad$ pp: 46-53. DOI: 10.1109/CVPR.2003.1211451 